\title{
Chemical Synthesis and Characterization of Poly(phenylhydrazine-co-4,4'-diaminodiphenyl Sulphone)
}

\author{
D. ILANGESWARAN ${ }^{*}$ and G. LAKSHMI \\ Department of Chemistry, Rajah Serfoji Govt. College (Autonomous), \\ Thanjavur -613005, India \\ dhailangeswaran@gmail.com
}

Received 15 December 2014 / Accepted 29 December 2014

\begin{abstract}
A new copolymer with nanostructure was chemically synthesized using an oxidizing agent between phenyl hydrazine hydrochloride (PH.HCl) and biologically active 4,4'-diaminodiphenyl sulfone (DADPS). The copolymer showed considerable solubility in common organic solvents such as chloroform, dimethyl formamide (DMF), dimethyl sulphoxide (DMSO) and tetrahydrofuran (THF). The copolymer showed electrical conductivity of $2.14 \times 10^{-2} \mathrm{~S} \mathrm{~cm}^{-1}$. The copolymer formation between these two monomers was confirmed by Fourier Transformation IR, ${ }^{1} \mathrm{H}$ NMR spectral studies, X-ray diffraction studies and scanning electron microscopic (SEM) data. The UV-Visible spectrum also showed the presence of benzene and NH units in the copolymer.
\end{abstract}

Keywords: Copolymer, Phenyl hydrazine, FTIR, NMR, XRD, SEM

\section{Introduction}

Copolymerization is a simple way of preparation for new polymers which greatly increases the scope of tailor-making materials with specifically desired properties ${ }^{1}$. Polyaniline is widely used as materials in electrochemical devices such as lightweight batteries, lightemitting diodes (LEDs) ${ }^{2}$, optical and electronic devices ${ }^{3}$, capacitors ${ }^{4}$, electrochromic devices $^{5,6}$, etc. Polyaniline has high conductivity ${ }^{7}$ at ambient temperatures. The insolubility of polyaniline in common solvents is its great disadvantage. Introduction of alkyl substituents in the benzene ring increased its solubility ${ }^{8}$. The addition of anions as dopant and copolymerization with other monomers improved the solubility. Recently great attention has been paid to synthesize aniline based copolymers. The conductivity of self-doped polyaniline synthesized by chemical copolymerization of aniline with $O$-amino benzenesulfonic acid was reported to depend on $\mathrm{pH} 0$ to $\mathrm{pH} 3^{9}$.

The self-doped sulfonated polyaniline was prepared chemically through the copolymerization of $p$-aminodiphenylamine with $o$-aminobenzenesulfonic $\operatorname{acid}^{10}$. The chemical copolymerization of aniline with $\mathrm{N}$-methylaniline, $\mathrm{N}$-butylaniline and 2-(4aminophenyl)-6-methylbenzothiozole have also been reported ${ }^{11,12}$. Lukachova et al. reported the chemical copolymerization of aniline with $m$-aminobenzenesulfonic acid using ammonium 
persulfate as the oxidant ${ }^{13}$. The copolymer prepared in this manner showed improvements in solubility, conductivity and stability in organic solvents ${ }^{14}$. Copolymers of aniline and 3aminophenol derivatives with oligo(oxyethylene) side chains as novel water soluble conducting polymers were reported ${ }^{15}$. The phenyl-substituted and $\mathrm{N}$-substituted copolymers of polyaniline with disordered structure exhibited better solubility and decreased conductivity $^{16-18}$.

Usage of materials with biological importance for the preparation of newer polymeric compounds assumes importance in the present environment ${ }^{19}$. Aligned or ordered, otherwise called poled polyurea sulfone thin films having excellent transparency from near UV to visible region were prepared by carrying out additional polymerization of 1,4-phenylene diisocyanate and 4,4'-diaminodiphenyl sulfone simultaneously. Electrochemically synthesized copolymer of aniline (ANI) and 4,4'-diaminodiphenyl sulfone (DADPS ${ }^{20}$ exhibited novel electrochromic properties. Copolymers of diphenylamine (DPA) and 4-aminodiphenylamine (ADPA) with 4,4'-diaminodiphenyl sulfone (DADPS) were prepared $^{21,22}$ characterized and their electrochromic properties also evaluated.

In this article, the chemical copolymerization of phenyl hydrazine (PH) with 4,4'-diaminodiphenyl sulfone (DADPS) using an oxidizing agent is presented. Solubility of the copolymers in various organic solvents and conductivities were studied. The copolymer formation was supported by FTIR, ${ }^{1} \mathrm{H}$ NMR, UV-visible spectra, XRD and SEM studies and the results are discussed.

\section{Experimental}

Reagent grade phenyl hydrazine hydrochloride (E-Merck) and potassium persulphate (E-Merck) were used as received. The other monomer 4,4'-diaminodiphenyl sulfone (DADPS) was synthesized ${ }^{23}$ by reacting thionyl chloride (reagent grade obtained from Zigma-Aldrich) with acetanilide (reagent grade obtained from Zigma-Aldrich), followed by oxidation to the sulfone with $\mathrm{CrO}_{3}$ (reagent grade obtained from Zigma-Aldrich). DADPS was then recrystallized to white crystals (m.p. $178-179{ }^{\circ} \mathrm{C}$ ) using ethanol. All solutions were prepared using ultra pure double distilled water.

\section{Chemical polymerization}

Phenyl hydrazine hydrochloride (PH.HCl) with 4,4'-diaminodiphenyl sulfone (DADPS) was polymerized by chemical oxidative method, using potassium persulphate as oxidizing agent in $1 \mathrm{M} \mathrm{HCl}$ medium. DADPS of $0.05 \mathrm{M}$ with $0.05 \mathrm{M}$ of PH. $\mathrm{HCl}$ in $250 \mathrm{~mL}$ of $1 \mathrm{M} \mathrm{HCl}$ having $15 \mathrm{~g}$ of potassium persulphate were stirred for $30 \mathrm{~min}$ in an ice bath by controlling the temperature between $0 \pm 5{ }^{\circ} \mathrm{C}$. After stirring for $10 \mathrm{~h}$ at room temperature, the polymer was precipitated with ammonia, centrifuged repeatedly, washed with ultrapure water followed by methanol and dried in vacuum at about $45^{\circ} \mathrm{C}$ for more than $12 \mathrm{~h}$.

\section{Characterization of chemically synthesized copolymer}

The solubility of copolymer, poly(phenyl hydrazine-co-4,4'-diaminodiphenyl sulfone) was tested in various organic solvents. The conductivity of this poly(PH-co-DADPS) was observed using four probe conductivity meter on pressed polymer pellets. UV-visible spectral studies were performed in a quartz cuvette with a path length of $1 \mathrm{~cm}$ utilizing a computer controlled JASCO V-530 UV-Visible spectrophotometer. The synthesized copolymer was characterized by FTIR spectral data recorded using $\mathrm{KBr}$-copolymer pellets on a SHIMADZU 8400S spectrophotometer. The polymers also characterized by ${ }^{1} \mathrm{H}$ NMR data obtained using BRUKER $300 \mathrm{MHz}$ NMR spectrometer. The surface morphology of the 
polymer films was studied utilizing SEM images obtained from a Hitachi S3000 H SEM instrument. The grain size of the copolymer was measured using XRD data obtained from an XRERT PRO PANALYTICAL instrument using $\mathrm{Cu} \mathrm{K}_{\alpha}$ radiation with $\lambda=1.5418 \AA$.

\section{Results and Discussion}

\section{Solubility of copolymers}

The solubility of the copolymer was tested with various organic solvents. The copolymer, poly(PH-co-DADPS) showed considerable solubility in dimethyl sulfoxide (DMSO), $N, N$-dimethyl formamide (DMF), tetrahydro furan (THF) and chloroform. The presence of amino groups as a side chain in the back bone of polymer may render the solubility in most of organic solvents.

\section{Conductivity studies of copolymers}

The electrical conductivity of pressed pellets of copolymer was measured, through four probe conductivity meter and it showed the conductivity of $2.14 \times 10^{-2} \mathrm{~S} \mathrm{~cm}^{-1}$. Compared to the conductivity of poly(4,4'-diaminodiphenyl sulfone $)^{19}$ slightly higher values are found for this copolymer.

\section{FTIR spectral behavior of copolymers}

FTIR spectral studies of the copolymers and homopolymers were carried out. The FTIR spectrum of poly4,4'-diaminodiphenyl sulfone (PDADPS) was already discussed ${ }^{21}$. In Figure 1, (A) and (B) represented the FTIR spectra of polyphenylhydrazine (PPH) and poly(PH-Co-DADPS) respectively. The vibrational frequencies observed for PDAPS and $\mathrm{PPH}$ along with that of copolymer, $\mathrm{P}(\mathrm{PH}-\mathrm{co}-\mathrm{DADPS})$ were presented in Table 1.

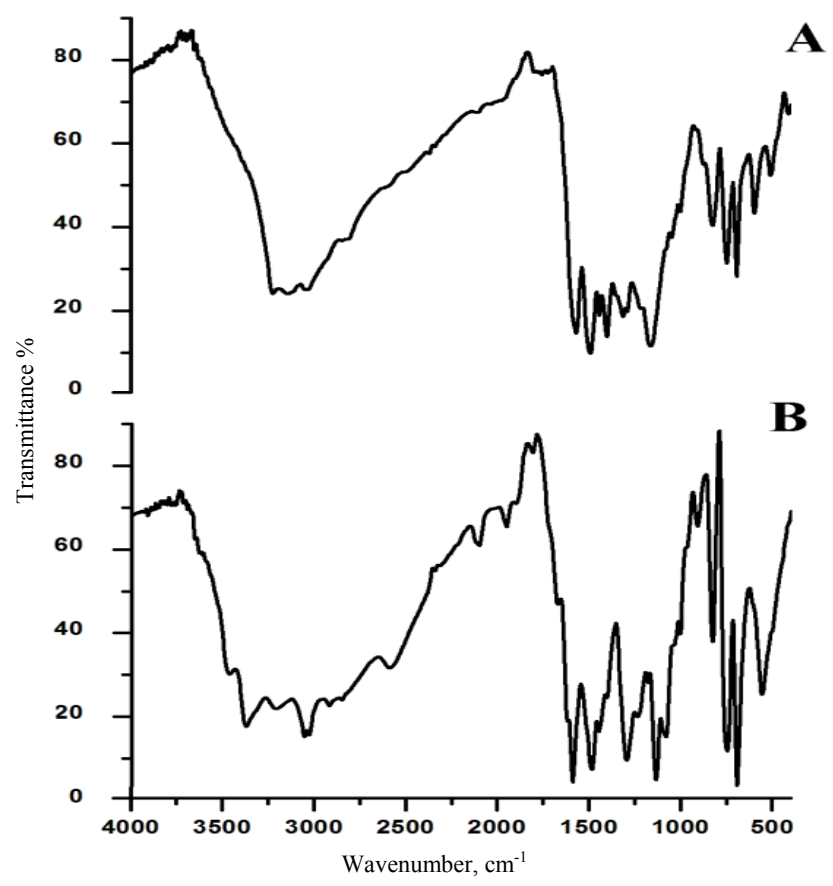

Figure 1. FTIR spectrum of (A) PPH and (B) P(PH-co-DADPS) 
Table 1. FTIR Spectral data of PDADS, PPH and P(PH-co-DADPS)

\begin{tabular}{|c|c|c|c|c|}
\hline \multirow{3}{*}{ Modes of vibrations } & \multirow{3}{*}{$\begin{array}{l}\text { Expected } \\
\text { wave number, } \\
\mathrm{cm}^{-1}\end{array}$} & \multicolumn{3}{|c|}{ Observed wave number, $\mathrm{cm}^{-1}$} \\
\hline & & \multicolumn{2}{|c|}{ Homopolymers } & \multirow{2}{*}{$\begin{array}{c}\text { Copolymer } \\
\text { P(PH-Co- } \\
\text { DADPS }) \\
\end{array}$} \\
\hline & & PDAPS & PPH & \\
\hline Secondary $(\mathrm{N}-\mathrm{H})_{\mathrm{s}}$ & $3300-3400$ & 3369 & 3225 & 3373 \\
\hline Secondary $(\mathrm{N}-\mathrm{H})_{\mathrm{b}}$ & $1550-1650$ & 1627 & - & - \\
\hline$(\mathrm{N}-\mathrm{H})_{\mathrm{s}}$ of $\mathrm{NH}_{2}$ group & $3400-3500$ & 3464 & - & 3464 \\
\hline$(\mathrm{N}-\mathrm{N})_{\mathrm{s}}$ & 1050 & 1015 & 1001 & 1084 \\
\hline$(\mathrm{N}-\mathrm{N})_{\mathrm{b}}$ & 660 & 648 & 597 & 696 \\
\hline$(\mathrm{N}=\mathrm{N})_{\mathrm{s}}$ & $1575-1630$ & 1591 & 1570 & 1593 \\
\hline$(\mathrm{C}=\mathrm{C})_{\mathrm{s}}$ & $1400-1600$ & 1427 & 1402 & 1448 \\
\hline$(\mathrm{C}-\mathrm{N})_{\mathrm{s}}$ & $1000-1250$ & 1149 & 1163 & 1138 \\
\hline$(\mathrm{C}-\mathrm{H})$ in plane bending & $1000-1300$ & 1103 & 1049 & 1138 \\
\hline$(\mathrm{C}-\mathrm{H})$ out of plane bending & $690-900$ & 686 & 694 & 748 \\
\hline $\begin{array}{l}(\mathrm{C}-\mathrm{H}) \text { out of plane bending of } \\
1,4 \text {-disubstituted benzene ring }\end{array}$ & $800-850$ & 837 & 823 & 829 \\
\hline$(\mathrm{S}=\mathrm{O})_{\mathrm{s}}$ & $\sim 1300$ & 1302 & - & 1298 \\
\hline
\end{tabular}

The presence of secondary N-H stretching frequency of PDADPS, $\mathrm{PPH}$ and $\mathrm{P}(\mathrm{PH}-\mathrm{co}-$ DADPS) at 3369,3225 and $3373 \mathrm{~cm}^{-1}$ respectively confirmed the $-\mathrm{NH}$ - units in the polymer chain. The frequency of $3464 \mathrm{~cm}^{-1}$ corresponding to the $\mathrm{N}-\mathrm{H}$ stretching of $\mathrm{NH}_{2}$ group was observed for $\mathrm{P}\left(\mathrm{PH}-\mathrm{co}\right.$-DADPS) showed that this copolymer contain $-\mathrm{NH}_{2}$ units in its back bone. The N-N stretching frequencies ${ }^{24}$ are clearly observed at 1015, 1001 and $1084 \mathrm{~cm}^{-1}$ for PDADPS, PPH and P(PH-Co-DADPS) respectively. Therefore it was supported that during homo and copolymerization processes the aromatic benzene rings of DADPS and PH are inter connected by $-\mathrm{N}-\mathrm{N}$ - linkages. Further the presence of sulfone group in the copolymer was evidenced by a sharp peak at $1298 \mathrm{~cm}^{-1}$ due to $\mathrm{S}=\mathrm{O}$ stretching vibration. The presence of free $-\mathrm{NH}_{2}$ group and $-\mathrm{N}-\mathrm{N}$ - linkages in the copolymer, $\mathrm{P}(\mathrm{PH}-\mathrm{co}-\mathrm{DADPS})$ by the IR spectra claimed that the phenyl hydrazine must be joined to DADPS as shown in Scheme 1.<smiles>Cc1ccc(S(=O)(=O)c2ccc(N(N)Nc3ccc(NN(N)Nc4ccc(S(=O)(=O)c5ccc(NC(C)(C)C)cc5)cc4)cc3)cc2)cc1</smiles>

Scheme 1. Proposed Structure of poly(PH-co-DADPS)

\section{${ }^{1} H$ NMR spectral studies of copolymers}

The proton NMR spectra for PPH and P(PH-co-DADPS) dissolved in $\mathrm{CDCl}_{3}$ are presented in Figures 2 and 3 respectively. The ${ }^{1} \mathrm{H}$ NMR spectrum of PDADPS was already reported and discussed ${ }^{21}$. A broad peak at $4.8 \mathrm{ppm}$ was noticed in the ${ }^{1} \mathrm{H}$ NMR spectrum of PPH given in Figure 2 and this correspond to the $\mathrm{NH}$ protons that might seen in the intermediate positions between various benzene units of polymer chain. The signals of aromatic (benzene/quinone) protons of PPH were observed as a multiplet between 7.030 to 7.947 $\mathrm{ppm}^{25}$. A small peak seen at $8.7 \mathrm{ppm}$ was due to $\mathrm{N}=\mathrm{NH}^{+}$protons of $\mathrm{PPH}$ in its polaronic / bipolaronic forms as given in Scheme 2.

The proton NMR spectrum of copolymer, $\mathrm{P}(\mathrm{PH}-\mathrm{co}-\mathrm{DPA})$ given in Figure 3 showed a group of three multiplet peaks between 7.221 to $7.758 \mathrm{ppm}$. These peaks were due to three different types of benzenoid/quinoid protons as seen in the Scheme 3 belong to both $\mathrm{PH}$ and DADPS monomer units in the polymer back bone. 


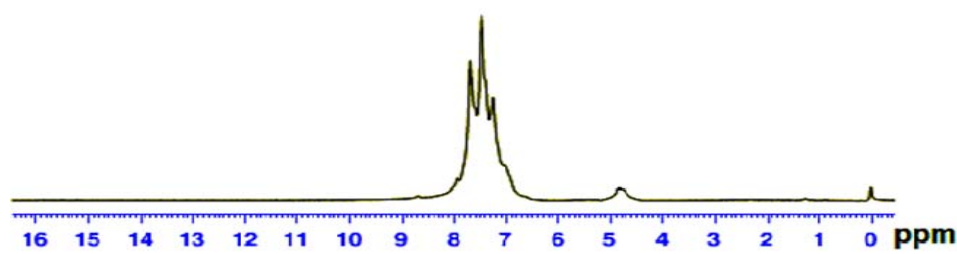

Figure 2. ${ }^{1} \mathrm{H}$ NMR spectrum of polyphenyl hydrazine in $\mathrm{CDCl}_{3}$

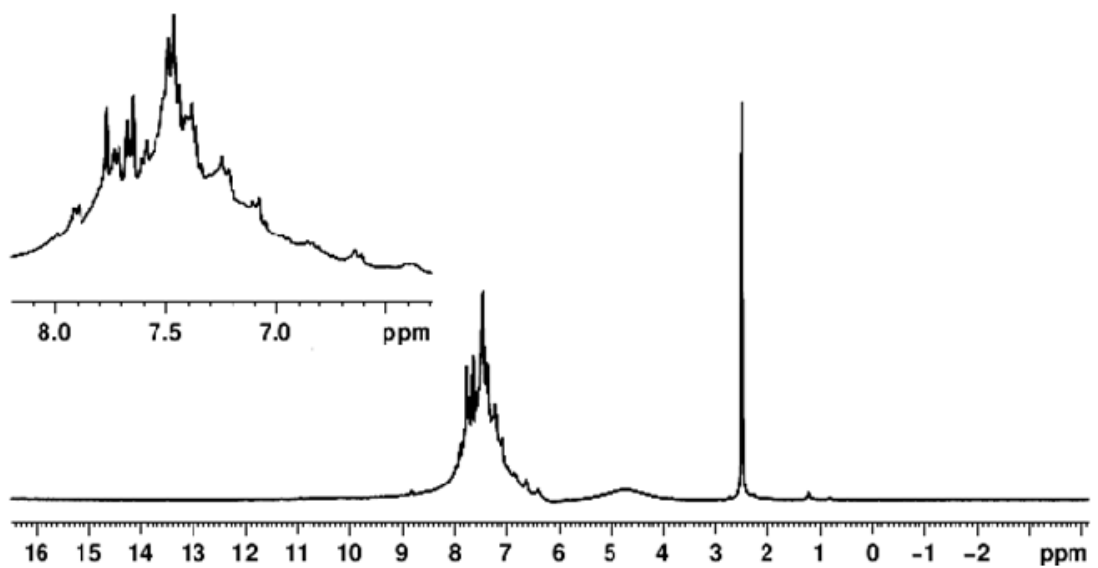

Figure 3. ${ }^{1} \mathrm{H}$ NMR spectrum of poly(phenylhydrazine-co-4,4'-diaminodiphenyl sulfone) in $\mathrm{CDCl}_{3}$.

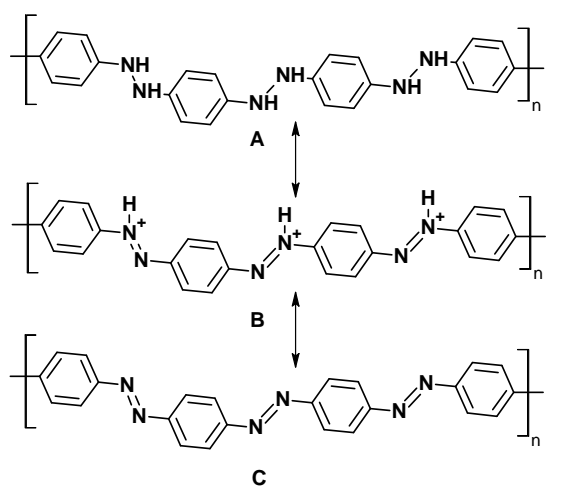

Scheme 2. (A) Reduced PPH, (B) polaronic/bipolaronic PPH and (C) Oxidized PPH

The signals for two types of $\mathrm{NH}$ protons as seen in the Scheme 3 were appearing at 7.155 and $7.081 \mathrm{ppm}$. The $-\mathrm{NH}_{2}$ protons present as a side chain on the polymer gave its signal at $4.8 \mathrm{ppm}$ as a broad peak. Most of the coupling constants measured for these proton signals existing between 6 and $10 \mathrm{~Hz}$ and this fact confirmed the coupling between ortho protons of benzene ring. This is possible only in the case of para disubstituted benzene rings. Thus most of the benzene units present in the polymer chain were 1,4-disubstituted and the structure for poly(PH-co-DADPS) as proposed in the above Scheme 3 was supported. The signals for $-\mathrm{NH}_{2}$ protons attached to $-\mathrm{N}=\mathrm{N}^{+}$- unit of the copolymer as shown in one of the resonance form of Scheme 3 were appeared at $8.9 \mathrm{ppm}$. 


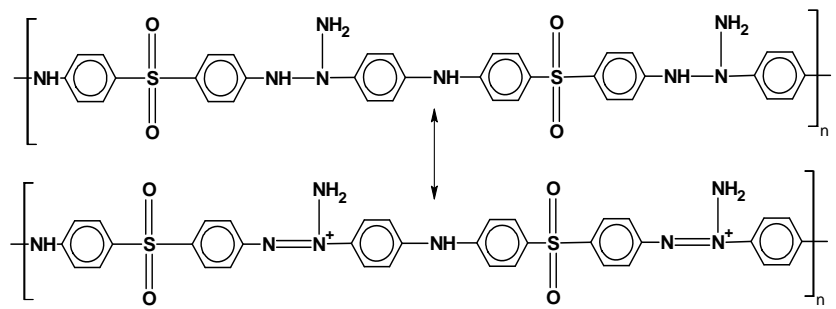

Scheme 3. Proposed structure for poly(PH-co-DADPS)

\section{XRD studies of copolymers}

The crystalline regions in the copolymers are shown by the presence of relatively sharp peaks. The amorphous regions are visible by the broad low intensity peaks. X-ray diffraction profile of the PPH and $\mathrm{P}(\mathrm{PH}-\mathrm{co}-\mathrm{DADPS})$ are shown as $\mathrm{A}$ and $\mathrm{B}$ in Figure 4. The XRD profile of PDAPPS was already discussed ${ }^{21}$. The homopolymer PPH showed lesser amorphous and more crystalline nature obviously due to the presence of sharp peaks. The XRD pattern of $\mathrm{P}(\mathrm{PH}-\mathrm{CO}-\mathrm{DADPS})$ indicated substantial increase in degree of amorphous nature evidenced the formation of copolymer. The particle size ${ }^{26-28}$ of homopolymer and copolymer were calculated from XRD studies using the Scherrer's formula as follows.

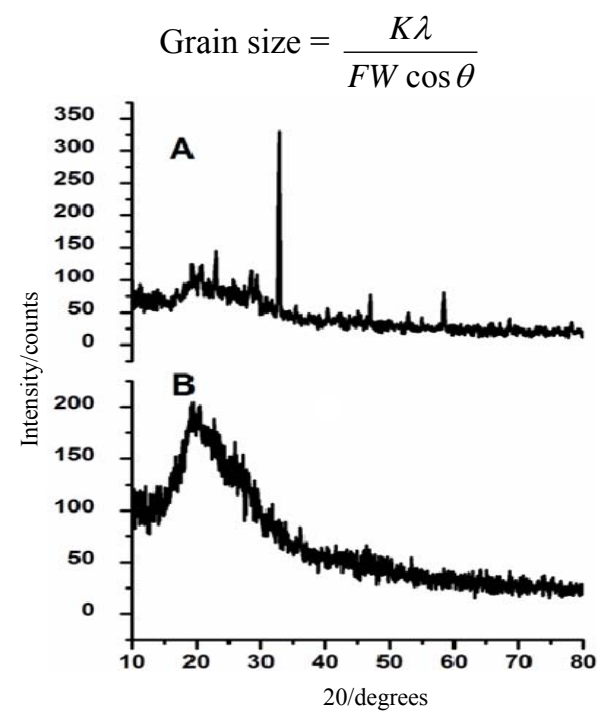

Figure 4. XRD pattern of (A) Poly(PH) and (B) Poly(PH-co-DADPS)

Where $\mathrm{K}$ is the shape factor of the average particle (expected to be 0.94 ), $\lambda$ is the wave length (usually $1.5418 \mathrm{~A}^{\circ}$ ), $\theta$ is the peak position and $\mathrm{FW}$ is the full width at half maximum. Using this formula the grain sizes of PPH and P(PH-Co-DADPS) were found to be 97 and 98 $\mathrm{nm}$ respectively. These facts evidenced the presence of nano structured copolymers.

\section{SEM Behaviour of copolymers}

Chemically copolymerized materials were characterized by scanning electron microscopic (SEM) analysis. SEM photographs of chemically synthesized homopolymers, PDADPS and $\mathrm{PPH}$ are presented in Figures 5 and 6 respectively. The SEM image of PDADPS appeared like a gathering of dried leaves while that of $\mathrm{PPH}$ showed spongy buds like structure in the 
micro meter scales respectively. The copolymer formed given in Figure 7 showed different structures in the form of scaled tiles like arrangement. This fact provides concrete evidence that newer polymeric materials are formed between the monomers and PH and DADPS.

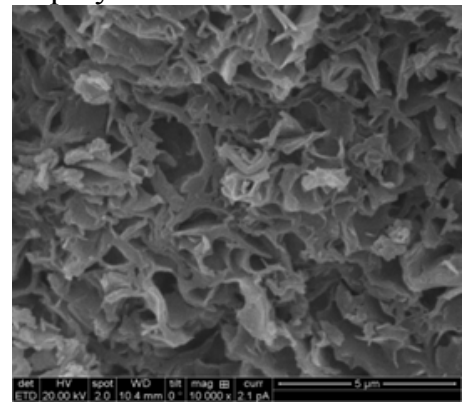

Figure 5. Scanning electron microscope photograph of PDADPS surface

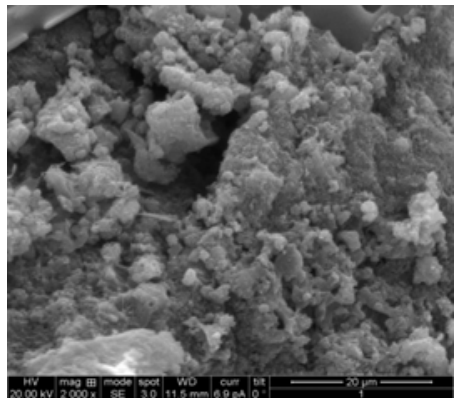

Figure 6. Scanning electron microscope photograph of PPH surface

\section{UV-VIS spectra of copolymers}

The UV-Visible spectral studies were carried out for copolymer, poly(PH-co-DADPS) in DMF and the spectrum was shown in Figure 8. Peaks with wavelength maximum at 280 and $350 \mathrm{~nm}$ were observed for the copolymer in DMF. These peaks may be associated with $\pi-\pi^{*}$ transition and conjugated benzenoid rings. This confirmed the presence of benzene rings in poly(PH-co-DADPS) as in phenylhydrazine and 4,4'-diaminodiphenyl sulfone. Another absorption band observed at $550 \mathrm{~nm}$ in copolymer was due to the polaron/bipolaron band transition. This band may be responsible for the dark brown color of the copolymer.

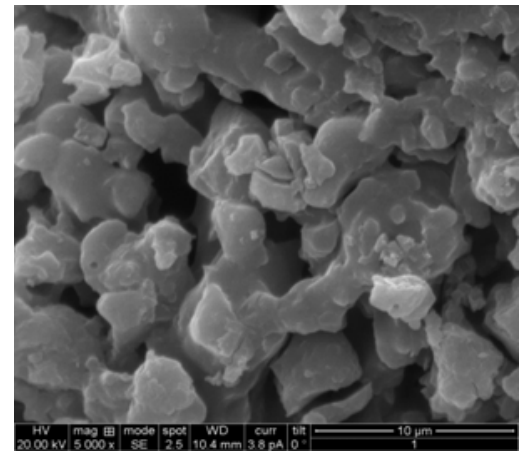

Figure 7. Scanning electron microscope photograph of $\mathrm{P}(\mathrm{PH}-\mathrm{co}-\mathrm{DADPS})$ surface

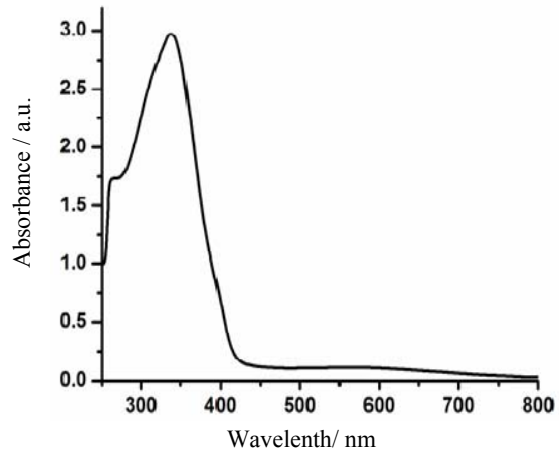

Figure 8. The UV-visible spectrum of poly(DPA-co-PH) in DMF

\section{Conclusion}

The various methods of characterization on the copolymers of phenyl hydrazine $(\mathrm{PH})$ and 4,4'-diaminodiphenyl sulfone synthesized using an oxidizing agent have been explained. Characterization of the copolymers by this host of techniques supports their copolymerization. These copolymers exhibited conductivity as well as solubility in common organic solvents. The copolymer formation and characteristics of functional groups were confirmed through FTIR and proton NMR spectral studies. The surface morphology and grain size were understood from SEM experiments. The XRD studies also evidenced the formation of micro sized copolymers. 


\section{References}

1 Bagheri A, Nateghi M R and Massoumi A, Synth Met., 1998, 97(2), 85-89; DOI:10.1016/S0379-6779(98)00090-3

2 Ho P K H, Kim J S, Burroughes J H, Becker H, Li S F Y, Brown T M, Cacialli F and Friend R H, Nature, 2000, 404(6777), 481; DOI:10.1038/35006610

3 Stucky G D, Nature. 2001, 410(6831), 885; DOI:10.1038/35073733

4 Moller S, Perlov C, Jackson W, Taussig C and Forrest S R, Nature, 2003, 426(6963), 166; DOI:10.1038/nature02070

5 Boehme J L, Mudigonda D S K and Ferraris J P, Chem Mater., 2001, 13(12), 44694472; DOI:10.1021/cm010346o

6 Argun A A, Aubert P H, Thompson B C, Schwendeman I, Gaupp C L, Hwang J, Pinto N J, Tanner D B, MacDiarmid A G and Reynolds J R, Chem Mater., 2004, 16(23), 4401-4412; DOI:10.1021/cm0496691

7 Han C C and Hong S P, Macromolecules, 2001, 34(14), 4937-4941; DOI: $10.1021 / \mathrm{ma} 002137 \mathrm{f}$

8 Cihaner A and Onal A M, Eur Polym J., 2001, 37(9), 1767-1772; DOI:10.1016/S0014-3057(01)00062-3

$9 \quad$ Fan J, Wan M and Zhu D, J Polym Sci Part A: Polym Chem., 1998, 36(17), 30133019; DOI:10.1002/(SICI)1099-0518(199812)36:17<3013::AID-POLA2>3.0.CO;2-W

10 Ohno N, Wang H J, Yan H and Toshima N, Polym J., 2001, 33(2), 165-171; DOI:10.1295/polymj.33.165

11 Langer J J, Synth Met., 1990, 35(3), 295-300; DOI:10.1016/0379-6779(90)90213-5

12 Abdelazzem M, Elhamouly S H and Hathoot A A, Eur Polym J., 1995, 31(12), 1207 1213; DOI:10.1016/0014-3057(95)00073-9

13 Lukachova L V, Shkerin E A, Puganova E A, Karyakina E E, Kiseleva S G, Orlov A V, Karpacheva G P and Karyakin A A, J Electroanal Chem., 2003, 544, 59-63; DOI:10.1016/S0022-0728(03)00065-2

14 Mu S, Synth Met., 2004, 143(3), 259-268; DOI:10.1016/j.synthmet.2003.12.008

15 Hua F and Ruckenstein E, Macromolecules, 2004, 37(16), 6104-6112; DOI:10.1021/ma040070g

16 Kumar Ram M, Maccioni E and Nicolini C, Thin Solid Films, 1997, 303(1-2), 27-33; DOI:10.1016/S0040-6090(97)00068-0

17 Ito A, Ota K, Tanaka K and Yamabe T, Macromolecules, 1995, 28(16), 5618-5625; DOI:10.1021/ma00120a029

18 Chavalier J W, Guay J and Dao L H, Macromolecules, 1992, 25(13), 3325-3331; DOI:10.1021/ma00039a001

19 Manisankar P, Vedhi C and Selvanathan G, J Polym Sci Part A: Polym Chem., 2005, 43(8), 1702-1707; DOI:10.1002/pola.20643

20 Manisankar P, Vedhi C, Selvanathan G and Somasundaram R M, Chem Mater., 2005, 17(7), 1722-1727; DOI:10.1021/cm0483700

21 Manisankar P and Ilangeswaran D, Electrochimica Acta, 2010, 55(22), 6546-6552; DOI:10.1016/j.electacta.2010.06.023

22 Ilangeswaran D and Manisankar P, Electrochimica Acta, 2013, 87, 895-904; DOI:10.1016/j.electacta.2012.09.040

23 Manisankar P, Sarpudeen A and Viswanathan S, J Pharm Biomed Anal., 2001, 26(56), 873-881; DOI:10.1016/S0731-7085(01)00480-0 
24 Mistry B D, A Handbook of Spectroscopic Data Chemistry, Oxford Book Company, India, 2009.

25 Shaolin Mu, Electrochimica Acta, 2014, 144, 243-253; DOI:10.1016/j.electacta.2014.08.020

26 Alexander L E, Diffraction Methods in Polymer Science, Wiley-Interscience, New York, 1969, 582.

27 Cai J, Yu Q, Zhang X, Lin J and Jiang L, J Poly Sci Part B: Polym Phys., 2005, 43, 2885-2897; DOI:10.1002/polb.20571

28 Fan Q, Ugbolue S C, Wilson A R, Mani G and Yang Y, "Dyeable Polypropylene via Nanotechnolgy", NTC Annual Report Nov, 2003, http://www.ntcresearch.org/pdfrpts/AnRp03/C01-MD20-A3.pdf. 